\section{LESSON 14}

MARGINS:

Left, Pica 20; Elite 30;

Right, moved out of the way.

PAPER: Against the paper guide at 0 on the scale on the left. Turn up 7 single lines.

Check the 'ready-to-type' position: feet, body, fingers and eyes.

\section{Warm up}

Return the carriage smartly. Keep your eyes on the copy. Tap the keys sharply.

\section{Figure 4}

Practise the reach until you can type 4 with the $f$ finger without looking down.

Keep the a finger in position.

Figure 7

Practise the reach until you can type 7 with the $\mathrm{j}$ finger without looking down.

Keep the ; finger in position.

\section{Figure 5}

Practise the reach until you can type 5 with the $f$ finger without looking down.

Keep the a finger in position.

\section{Figure 6}

Practise the reach until you can type 6 with the $\mathrm{j}$ finger without looking down.

Keep the ; finger in position.

\section{Consolidation}

Check the 'ready-to-type' position.

Use the paper release lever when taking the paper out of the machine.

Centre and lock the carriage.

Cover the machine.

\section{UNIT 4}

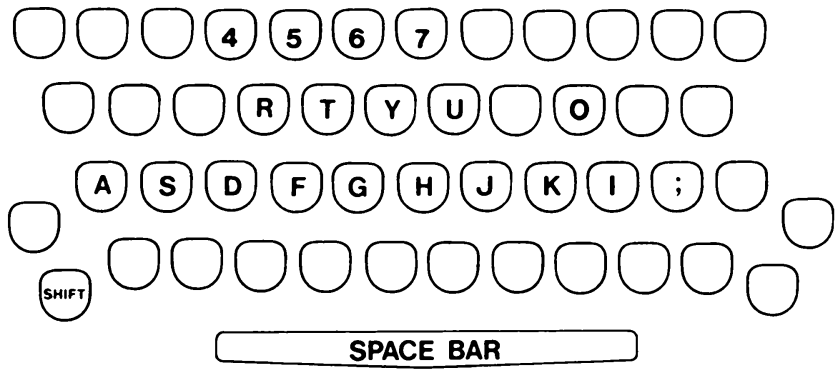

Remember to leave a blank line between sections.

Type each pair of lines 3 times and try to build up speed.

In the lessons which follow, you will learn how to type the numbers along the top row of the machine.

Some of you will see that you do not have either a figure one or a nought on your typewriter, and you must use a small $l$ and a capital 0 .

Type the first line of each new figure as many times as your teacher asks.

Type the other lines indicated by your teacher 3 times each.

1. fff frf fr4 fr4 444 f $44 \mathrm{f}$ fr 4 4rf $r 4 f$ f $4 \mathrm{f}$

2. 4 and 4 and $O$ less 4 and 4 and $O$ makes $O$

3. The 4 boys and 4 girls met, 4 weeks ago.

4. jjj juj ju7 ju7 777 ju7 j7u7 ju7 ju7 j7j

5. 7 and 7 and $O$ less 7 and 7 and $O$ makes $O$

6. The 7 teams met 7 times in just 7 weeks.

7. fff ftf ft5 ft5 555 f5t5 f5f t5t ft5 f5f

8. 5 plus 5 is $10 ; 5$ plus 5 plus 5 makes 15

9. See 55 join the 500 to make 555 campers.

10. jjј jyj jy6 jy6 666 j6j6 y6y jy6 jy6 j6j

11. 6 and 6 and 0 less 6 and 6 and 0 makes 0 12. The 6 men hit 60 runs to make 66 in all.

Type each line 3 times.

13. See the 4 th, 5 th, 6 th and 7 th men alone.

14. Take 145 from 167 and divide the answer.

15. The 45 boys and 56 girls saw 71 animals.

Messieurs is abbreviated to Messrs. What are the abbreviations for Esquire, Mister and Mistress?

Why is Miss not followed by a full stop? 